\title{
The Effectiveness of Thai Massage and Joint Mobilization
}

\author{
Chantip Juntakarn, $\mathrm{MA}^{3}$ Thavat Prasartritha, MD, ${ }^{1}$ Prapoj Petrakard, $\mathrm{MD}^{2}$ \\ ${ }^{1}$ Institute of Orthopaedics, Lerdsin Hospital, Bangkok, Thailand, ${ }^{2}$ Department of Thai Traditional and Alternative Medicine, \\ Ministry of Public Health, Nonthaburi, Thailand, ${ }^{3}$ Department of Academic Support, Lerdsin Hospital, Bangkok, Thailand
}

Background: Non-specific low back pain (LBP) is a common health problem resulting from many risk factors and human behaviors. Some of these may interact synergistically and have been implicated in the cause of low back pain. Massage both traditional Thai massage and joint mobilization as a common practice has been shown to be effective for some subgroup of nonspecific LBP patients.

Purpose and Setting: The trial compared the effectiveness between traditional Thai massage and joint mobilization for treating nonspecific LBP. Some associated factors were included. The study was conducted at the orthopedic outpatient department, Lerdsin General Hospital, Bangkok, Thailand.

Methods: Prospective, randomized study was developed without control group. The required sample size was estimated based on previous comparative studies for effectiveness between techniques. Two primary outcome measures were a 0 to 10 visual analog scale (VAS) of pain and Oswestry Disability Index (ODI). Secondary outcome measures were satisfaction of patients and adverse effects of the treatment. The "intention to treat" (ITT) and per protocol approach were used to compare the significance of the difference between treatment groups

Participants: One hundred and twenty hospital outpatients, $20(16.7 \%)$ male and $100(83.3 \%)$ female, were randomized into traditional Thai massage and joint mobilization therapy. The average age of traditional Thai massage and joint mobilization was 50.7 years and 48.3 years, respectively. Both groups received each treatment for approximately 30 minutes twice per week over a four-week period. Total course did not exceed eight sessions.

Result: With ITT, the mean VAS of traditional Thai massage group before treatment was 5.3 (SD $=1.7)$ and ODI was $24.9(\mathrm{SD}=14.7)$, while in joint mobilization groups, the mean VAS was 5.0 (SD $=1.6)$ and ODI was 24.6 $(\mathrm{SD}=15)$. After treatment, the mean VAS and ODI were significantly reduced $(\mathrm{VAS}=\mathbf{0 . 5 1}(\mathrm{SD}=\mathbf{0 . 8 9})$ and $\mathrm{ODI}=\mathbf{8 . 1}$ $(\mathrm{SD}=10.7)$ for traditional Thai massage, VAS $=$
$0.86(\mathrm{SD}=1.49)$ and $\mathrm{ODI}=\mathbf{8 . 2 6}(\mathrm{SD}=12.97)$ for joint mobilization). Constipation was found in 34 patients $(28.3 \%)$.

Conclusion: The traditional Thai massage and joint mobilization used in this study were equally effective for short-term reduction of pain and disability in patients with chronic nonspecific LBP. Both techniques were safe with short term effect in a chosen group of patients.

KEY WORDS: effectiveness; Thai massage; joint mobilization, low back pain

\section{INTRODUCTION}

Nonspecific low back pain (LBP) can be highly complex from a wide variety of risks and patients' own behaviors. In the literature, over 100 potential risks have been identified..$^{(1,2)}$ The impact of psychosocial factors both work and nonwork-related on the prevalence of LBP has been studied. (3) Though the evidence is inconclusive, some associations exist. Significantly, some may interact synergistically and have been implicated in the cause of work-related low back pain. ${ }^{(4)}$ The costly consequences, mainly due to excessive and inappropriate use of diagnoses and variety of treatment programs, have been referred to as an enormous burden on society, the health care system, and the economies of many countries. ${ }^{(5-7)}$

As pain is still the most important factor in nonspecific LBP, studies of the effectiveness of intervention to relieve pain are the main focus. Currently, there are more than 50 potential therapies promising to relieve pain and lessen disability. ${ }^{(7-9)}$ Of these, one of the oldest forms of treatment is soft tissue massage, a hand manipulation to reduce stress and pain. ${ }^{(10-13)}$ Practice of this therapy has increased worldwide for health reasons. Every country has its own styles and settings based on national traditions and cultures. However, evidence indicated that massage therapy might be effective in some subgroups of patients and should be performed by an experienced manipulator. ${ }^{(14-15)}$

Currently, massage practice has been considered as one of the fastest growing sectors of the 
complementary and alternative therapy in the health care systems in Thailand and overseas. ${ }^{(10-12)}$ Traditional Thai massage is one branch of Thai traditional medicine that originated in Thai society through the learning and development of drug formulas and arts for health care. ${ }^{(16)}$ There are two basic principles comprised of four primary elements (earth, water, fire, and air or wind) and the body's 10 main lines (sen prathan sib). Such main lines are the routes for transmitting inner energy through the body. The blockage or increase of wind flow through these lines will cause bodily pain or dysfunction. The 10 main lines have the centre underneath and around the umbilicus and are orderly distributed in all parts of the body. ${ }^{(15,17-18)}$ In practice, traditional Thai massage is a deep massage with prolonged pressure along the body's 10 major energy channels with passive gentle stretching that is believed to release the blocked energy, increasing awareness and vitality. ${ }^{(16,19)}$

Similar to other countries, the effects of traditional Thai massage have been shown to enhance health and well-being. ${ }^{(16-19)}$ In the past decade, there were few randomized controlled trials examining the effects of the massage. ${ }^{(15,20-21)}$ Findings showed potential risk of bias and global disparities in the studies. Recently, an updated systematic review has reported that massage might be beneficial to patients with subacute and chronic nonspecific LBP. ${ }^{(22-24)}$ But there has been a need for quality randomized trials on the assessment. The current trial was developed without control group to compare the effectiveness between traditional Thai massage and joint mobilization for treating nonspecific LBP. ${ }^{(25)}$ Joint mobilization or spinal manipulation was selected because it is commonly used or recommended in clinical setting. ${ }^{(9,11-13,22,23)}$ Outcomes of treatment groups were determined using methods appropriate for noninferiority intention-totreat analysis (ITT). ${ }^{(26)}$ Some associated risk factors were also reported.

The objective of this study was to compare the effectiveness of traditional Thai massage with joint mobilization. It was hypothesized that the two techniques are predictively equivalent in terms of immediate pain - VAS (Visual Analog Scale) and ODI (Oswestry Disability Index). Furthermore, a second null hypothesis was expected to demonstrate no difference between pretest and posttest scores in the treatments effect on pain and function.

\section{METHODS}

This prospective, randomized study was conducted at Lerdsin Hospital, Department of Medical Services, Ministry of Public Health, Bangkok, Thailand. Ethical approval was obtained from the Ethics Review Committee of the hospital. All participants were recruited at the orthopedic outpatient department between October 2010 and April 2011.

\section{Participants}

The eligibility criteria included patients with nonspecific LBP that was intermittent (symptoms may fluctuate from day to day ${ }^{(23-24)}$ ) and could be tolerated; the straight leg raising test was negative; the VAS was at least 3; and the duration of pain was more than three weeks. Patients were screened out if they had persistent or significant disability due to severe back pain and VAS of more than 7. All patients with severe systematic diseases (such as SLE, rheumatic disease, infection, malignancy), previous spine surgery, pregnancy, and psychiatric disease were excluded. All patients should be able to attend full course of therapy and provided a written informed consent. The information on rationale, and possible advantages and disadvantages in attending this study was given to each participant.

\section{Sample Size}

The required sample size was estimated based on previous comparative studies for effectiveness between techniques. Outcome reduction of 1 VAS was considered as significant difference between groups. ${ }^{(15,18)}$ Accordingly, at least 60 subjects were required in each group to detect 1-2 scales of VAS reduction following treatment with a significant level of 5\% and statistical power of $95 \%$. The dropout rate should not exceed $20 \%$.

\section{Randomization}

Patients were randomly assigned into two groups: traditional Thai massage and joint mobilization, using a computer-generated sequence of random numbers. The allocation was managed by an independent research assistant and not decoded until the intervention was assigned.

\section{Interventions}

Spinal manipulation or joint mobilization consisted of physical assessment, active and passive physiologic back mobilization stretching and strengthening, and thermo-therapy with hot pack. ${ }^{(14)}$ Posterior-toanterior (AP) directed manual pressures was applied to the spinous process of the lumbar vertebrae, as described by Maitland ${ }^{(27)}$ and Beattie et al., ${ }^{(28)}$ followed by the prone press-up exercise as described by McKenzie. ${ }^{(29)}$ The basic principle as proposed is the gate control theory - blocking central pain transmission by the increase of proprioceptive input. This simple concept may explain why rubbing an injured site would alleviate pain, as well as why mobilization can control pain after musculoskeletal trauma. ${ }^{(30)} \mathrm{A}$ common practice in physical therapy departments, joint manipulation has been shown to be effective for nonspecific and acute LBP. ${ }^{(31-32)}$

For traditional Thai massage, the key elements emphasize the safe performance and true relaxation 
of body and mind as routine before massage. ${ }^{(16)}$ Life energy (like a power wave) should be established and transmitted from the therapist through the patient's back. Enough pressure should be firmly applied and released slowly to keep the state of relaxation. The flow of the massaging movement should be slow and steady without disruption and maintained throughout the session. Massage was conducted by pressing and mobilizing points on two main energy lines (Ida and Pingala) which run along spinous processes from L2 to L5. Stretching and strengthening of specific back muscles, as well as herbal hot packs, were included.

Both forms of treatment were given by certified physical and massage therapists who had been working at the hospital and who had more than 10 years of experience. Both the therapist and the patient concentrated on the treatment with minimal verbal communication. Assessment of pain and back motion was routinely performed prior to treatment. Each group of therapists should adhere strictly to the treatment protocol, following exact steps stated in the manual of standard procedure to minimize differences in the components in each treatment. Both groups received two treatments per week over a four-week period. Total course should not exceed eight sessions. Every treatment in each group lasted approximately 30 mins.

Patients were asked not to use their own NSAIDs and analgesics. Only paracetamol and diclofenac were prescribed as a rescue drug for back pain during the trial in both groups. At home, all patients were encouraged to engage in general back plus leg stretching and strengthening exercises. ${ }^{(33)}$ Trial would be immediately terminated in cases where the physician believed that there was any unacceptable or uncontrollable risk of serious events.

\section{Statistical Method}

Mean, standard deviation (SD), and percentage were calculated for each demographic data. All data were analyzed using the SPSS statistic program. Parametric methods were used to calculate the statistical significance of VAS and ODI, paired $t$ test was used to compare outcome variables at baseline (before and after treatment session). The "intention to treat" and per protocol approach were used to compare the significance of the different between treatment groups. ${ }^{(26)}$ Improvement of $50 \%$ and $30 \%$ were rated as a threshold for success and minimal improvement, respectively. $(15,18)$

The baseline information included demographic data, body mass index, occupation, working hours, physical activity, smoking, alcohol use, sleeping hours and constipation. Two primary outcome measures were a 0 to 10 visual analog scale (VAS) of pain or bothersome symptoms and Oswestry Disability Index (ODI) for functionality. ${ }^{(34)}$ The ODI questionnaire contained 10 questions concerning functional deficit related to pain. Each question was scored from 0 to 5 , with higher values indicating more severe impact. Total scores were subsequently transformed into percentages $(0 \%-20 \%$ indicating minimal disability; $21 \%-40 \%$, moderate disability; $41 \%-60 \%$, severe disability; $61 \%-80 \%$, crippled; and $81 \%-100 \%$, bed-bound). Secondary outcome measures were satisfaction of patients and adverse effects of the treatment. All measures were assessed immediately before treatment and the 8th visits afterwards by one well-trained research supervisor who was unknown to each patient. Patients were rated as "satisfied", "lower satisfied", or "unsatisfied", using LBP outcome assessment tool. (35-36)

\section{RESULTS}

Of the 345 potential subjects, 120 met the inclusion criteria (20 males or $13.3 \%$ and 100 females or $86.7 \%$ ). The average BMI was $24.35 \mathrm{~kg} / \mathrm{m}^{2}$ (traditional Thai massage $=24.0$, joint mobilization $=24.7)$. Overall mean age $+\mathrm{SD}$ was $49.5+10$ yrs. The most common age range was 50-60 years. A comparison of all variables is shown in Table 1 . Insignificant differences among groups were noticed for occupation, working hours, types of work, physical activity, sleeping hours, and constipation.

TABLE 1. Demographic Data and Affecting Factors

\begin{tabular}{|c|c|c|c|}
\hline & $\begin{array}{c}\text { Traditional } \\
\text { Thai Massage }\end{array}$ & $\begin{array}{c}\text { Joint } \\
\text { Mobilization }\end{array}$ & Total \\
\hline \multicolumn{4}{|l|}{ Gender n (\%) } \\
\hline Male & $12(20)$ & $8(13.3)$ & $20(16.7)$ \\
\hline Female & $48(80)$ & $52(86.7)$ & $100(83.3)$ \\
\hline Age (yr.) & $50.7(9.8)$ & $48.3(10.2)$ & $49.48(10.0)$ \\
\hline \multicolumn{4}{|l|}{ Occupation n (\%) } \\
\hline labor & $13(21.7)$ & $21(35.0)$ & $34(28.3)$ \\
\hline office & $18(30.0)$ & $19(31.7)$ & $37(30.8)$ \\
\hline house wife & $23(38.3)$ & $11(18.3)$ & $34(28.3)$ \\
\hline vendor & $5(8.3)$ & $7(11.7)$ & $12(10.0)$ \\
\hline unemployed & $1(1.7)$ & $2(3.3)$ & $3(2.5)$ \\
\hline total & $60(100)$ & $60(100)$ & $120(100)$ \\
\hline \multicolumn{4}{|l|}{ Risky habits n (\%) } \\
\hline tobacco \& alcohol & 0 & 0 & 0 \\
\hline only tobacco & $2(3.3)$ & $2(3.3)$ & $4(3.33)$ \\
\hline only alcohol & $7(11.7)$ & $5(8.3)$ & $12(10.0)$ \\
\hline No tobacco \& alcohol & $51(85.0)$ & $53(88.3)$ & $104(86.7)$ \\
\hline total & $60(100)$ & $60(100)$ & $120(100)$ \\
\hline \multicolumn{4}{|l|}{ Sleeping hours $n(\%)$} \\
\hline$<6$ & $25(41.7)$ & $18(30.0)$ & $43(35.8)$ \\
\hline $6-8$ & $33(55.0)$ & $39(65.0)$ & $72(60.0)$ \\
\hline$>8$ & $2(3.3)$ & $3(5.0)$ & $5(4.2)$ \\
\hline total & $60(100)$ & $60(100)$ & $120(100)$ \\
\hline \multicolumn{4}{|l|}{ Constipation $n(\%)$} \\
\hline no & $47(78.3)$ & $39(65.0)$ & $86(71.7)$ \\
\hline yes & $13(21.7)$ & $21(35.0)$ & $34(28.3)$ \\
\hline total & $60(100)$ & $60(100)$ & $120(100)$ \\
\hline
\end{tabular}


Constipation was found in 34 patients (28.3\%). ODI in the patients with constipation was insignificantly greater than in those with nonconstipation (27.7 vs. 23.5). Regarding BMI, there was no significant difference between the two groups (Table $2)$. The ratio of patients with regular to nonregular exercise was 4.7: 1 (99 to 21 patients); less physical activity was found to be significantly related to less disability.

Four cases of traditional Thai massage and nine cases of joint mobilization dropped out before treatment due to inconvenience. Following the randomization, there were 60 patients in each group. The mean VAS and ODI in the traditional Thai massage group were slightly higher than in the joint mobilization group (Table 2). As shown in Table 3(a)(ITT) and

Table 2. Data Comparison Between Groups ${ }^{\mathrm{a}}$

\begin{tabular}{lccccc}
\hline & $\begin{array}{c}\text { Traditional } \\
\text { Thai } \\
\text { Massage }\end{array}$ & $\begin{array}{c}\text { Joint } \\
\text { Mobilization }\end{array}$ & $\begin{array}{c}\text { Mean } \\
\text { Diff. }\end{array}$ & P value & 95\%CI \\
& & & & & \\
\hline \multirow{2}{*}{ Age } & 50.7 & 48.3 & 2.33 & 0.204 & -1.282, \\
& $(9.8)$ & $(10.2)$ & $(1.83)$ & & 5.949 \\
BW (kg.) & 59.5 & 61.8 & -2.30 & 0.236 & -6.114, \\
& $(10.5)$ & $(10.6)$ & $(1.90)$ & & 1.524 \\
Ht (cm.) & 157.5 & 158.4 & -0.93 & 0.546 & -3.986 , \\
& $(8.2)$ & $(8.7)$ & $(1.54)$ & & 2.120 \\
BMI & 24.0 & 24.7 & -0.74 & 0.325 & -2.21, \\
& $(3.8)$ & $(4.3)$ & $(0.75)$ & & 0.741 \\
VAS (visit 0) & 5.3 & 5.0 & 0.35 & 0.247 & -0.25, \\
& $(1.7)$ & $(1.6)$ & $(0.30)$ & & 0.95 \\
ODI (visit 0) & 24.9 & 24.6 & 0.26 & 0.923 & -5.11, \\
& $(14.7)$ & $(15.0)$ & $(2.71)$ & & 5.63 \\
\hline
\end{tabular}

${ }^{a}$ No significance between groups

Table 3(a). Primary Outcomes Before and After Treatment: Compared Independent $t$ Test (Intention to Treat)

\begin{tabular}{|c|c|c|c|c|c|}
\hline & \multicolumn{2}{|c|}{ Mean (SD) } & \multirow{2}{*}{$\begin{array}{c}\text { Mean } \\
\text { Diff. }\end{array}$} & \multirow{2}{*}{$\begin{array}{c}p \\
\text { value }\end{array}$} & \multirow[t]{2}{*}{$95 \% \mathrm{Cl}$} \\
\hline & $\begin{array}{l}\text { Traditional } \\
\text { Thai Massage } \\
\quad(n=60)\end{array}$ & $\begin{array}{c}\text { Joint } \\
\text { Mobilization } \\
(n=60)\end{array}$ & & & \\
\hline \multicolumn{6}{|l|}{ VAS } \\
\hline Visit 0 & $\begin{array}{c}5.3 \\
(1.7)\end{array}$ & $\begin{array}{c}5.0 \\
(1.60)\end{array}$ & $\begin{array}{c}0.35 \\
(0.30)\end{array}$ & 0.247 & $\begin{array}{c}-0.245 \text {, } \\
0.945\end{array}$ \\
\hline Visit $8^{a}$ & $\begin{array}{c}0.513 \\
(0.886)\end{array}$ & $\begin{array}{l}0.857 \\
(1.49)\end{array}$ & $\begin{array}{l}-0.099 \\
(0.14)\end{array}$ & 0.483 & $\begin{array}{c}-0.378, \\
0.180\end{array}$ \\
\hline \multicolumn{6}{|l|}{ ODI } \\
\hline Visit 0 & $\begin{array}{l}24.852 \\
(14.66)\end{array}$ & $\begin{array}{c}24.589 \\
(15.040)\end{array}$ & $\begin{array}{c}0.26 \\
(2.71)\end{array}$ & 0.923 & $\begin{array}{c}-5.106 \text {, } \\
5.632\end{array}$ \\
\hline Visit $8^{a}$ & $\begin{array}{c}8.120 \\
(10.744)\end{array}$ & $\begin{array}{c}8.259 \\
(12.973)\end{array}$ & $\begin{array}{c}1.03 \\
(1.94)\end{array}$ & 0.597 & $\begin{array}{c}-2.81 \\
4.87\end{array}$ \\
\hline
\end{tabular}

${ }^{a}$ VAS and ODI significantly declined after treatment (visit 8)
Table 3(b)(per protocol), pain intensity (VAS) in both groups significantly declined after treatment, but the difference between groups was insignificant. The traditional Thai massage group had better scores in terms of pain reduction which lasted for one month. The joint mobilization group had better reduction in terms of functional disability. Final outcome measures of VAS and ODI were not significant between groups (Tables 3(c) and 3(d)). For secondary outcome measures, both groups were satisfied with the assigned treatment without any adverse event. Improvement was shown at the 8 th visits. Overall percentage improvement of ODI in the traditional Thai massage and joint mobilization groups were $67.1 \%$ and $66.3 \%$, respectively (Table $3(\mathrm{~d})$ ).

TABle 3(b). Primary Outcomes Before and After Treatment: Compared Independent $t$ Test (Protocol)

\begin{tabular}{|c|c|c|c|c|c|}
\hline & \multicolumn{2}{|c|}{ Mean (SD) } & \multirow{2}{*}{$\begin{array}{c}\text { Mean } \\
\text { Diff. }\end{array}$} & \multirow{2}{*}{$\begin{array}{c}p \\
\text { value }\end{array}$} & \multirow[t]{2}{*}{$95 \% \mathrm{CI}$} \\
\hline & $\begin{array}{l}\text { Traditional } \\
\text { Thai Massage } \\
\quad(n=56)\end{array}$ & $\begin{array}{c}\text { Joint } \\
\text { Mobilization } \\
(n=51)\end{array}$ & & & \\
\hline \multicolumn{6}{|l|}{ VAS } \\
\hline Visit 0 & $\begin{array}{c}5.32 \\
(1.716)\end{array}$ & $\begin{array}{c}4.808 \\
(1.594)\end{array}$ & $\begin{array}{c}0.51 \\
(032)\end{array}$ & 0.115 & $\begin{array}{c}-0.127 \\
1.147\end{array}$ \\
\hline Visit 8 & $\begin{array}{c}0.384 \\
(0.673)\end{array}$ & $\begin{array}{c}0.510 \\
(0.815)\end{array}$ & $\begin{array}{l}-0.13 \\
(0.14)\end{array}$ & 0.348 & $\begin{array}{c}-0.411- \\
0.160\end{array}$ \\
\hline \multicolumn{6}{|l|}{ ODI } \\
\hline Visit 0 & $\begin{array}{c}24.456 \\
(14.662)\end{array}$ & $\begin{array}{c}23.203 \\
(13.651)\end{array}$ & $\begin{array}{c}1.25 \\
(2.75)\end{array}$ & 0.649 & $\begin{array}{c}-4.192- \\
6.700\end{array}$ \\
\hline Visit 8 & $\begin{array}{c}7.432 \\
(10.246)\end{array}$ & $\begin{array}{c}6.780 \\
(10.239)\end{array}$ & $\begin{array}{c}0.65 \\
(1.98)\end{array}$ & 0.743 & $\begin{array}{c}-3.279- \\
4.583\end{array}$ \\
\hline
\end{tabular}

TABle 3(c). Primary Outcomes Before and After Treatment: Significance of Treatments (Pair's $t$ Test) ${ }^{\mathrm{a}}$

\begin{tabular}{lccc}
\hline & Mean Diff. & Sig. & $95 \% C I$ \\
\hline VAS (V0,V8) & $4.66(1.76)$ & $<.001$ & $4.33,5.00$ \\
ODI (V0,V8) & $16.81(12.6)$ & $<.001$ & $14.45,19.18$ \\
\hline
\end{tabular}

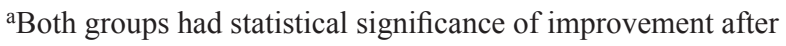
treatment $p<.001$

TABle 3(d). Primary Outcomes Before and After Treatment: Percentage $(\%)$ of Improvement by ODI Between Groups

\begin{tabular}{ccc}
$\begin{array}{c}\% O D I \\
(n=60)\end{array}$ & $\begin{array}{c}\text { Traditional } \\
\text { Thai Massage }\end{array}$ & $P T$ \\
\hline Visit 0-4 & 38.2 & 40.2 \\
Visit 4-8 & 46.8 & 43.5 \\
Visit 0-8 & 67.1 & 66.3 \\
\hline
\end{tabular}




\section{DISCUSSION}

The current randomized trial was carried out without control group. This can be a practical clinical trial or comparative effectiveness research (CER), which specifically compares two relevant alternative interventions in specific population. $(25,37)$ Under carefully controlled study, the obtained result can be considered as reliable evidence. Although CER has been defined and developed, the trials are not yet widely accepted as it would raise a number of challenging scientific and ethical issues. ${ }^{(37)}$ The near similarity at baseline of the current study groups may indicate the consistency of screening methodology. Although it was not the main objective, the study had focused on some lifestyle-affecting factors such as sleeping hours, physical exercise, smoking, and constipation. Various associated factors were in line with other reports.

Though overweight has been reported to induce less bowel activity, ${ }^{(38)}$ the study has found no statistically significant association between defecation and BMI. Further studies on the modification of lifestyle and the use of more effective palliative options are needed to assess their impact on the development of nonspecific LBP.

A better result of treating nonspecific LBP and associated disability has continuously been presented over time. However, delayed recovery within one year of treatment at a primary care unit is a common expectation. ${ }^{23,39)}$ Treatment effect as shown above suggested that all selected patients were most likely to have immediate benefit from traditional Thai massage or joint mobilization. Both methods are safe and demonstrated improvement with time. The current protocol had included self-stretching and strengthening exercises, which have been widely accepted. With this approach, patients would have sufficient knowledge and necessary skills to cope with their problems and to maintain adequate daily psychosocial function. (33) They should be encouraged to take their own active roles in self-management as a part of their lives.

In general, pain appears to be a common outcome measurement. With the findings, pain has been shown as a reliable responsive indicator correlated well with the ODI. Improvements in ODI are used to define a more relevant score change on each treatment group. According to Fritz et al., (32) this measure as a threshold for success should be applied at the level of an individual patient, but not to compare between groups. Even though the finding was unable to discriminate efficacy between traditional Thai massage and joint mobilization, the benefits from their effects could be expected in a chosen group of patients.

In accordance with previous studies, ${ }^{(17-18)}$ the findings support the use of short-term conservative methods in alleviating chronic LBP. However, it could not answer how long an effect may last following the termination of treatment. There were several limitations, namely: 1) the sample size was too small; in case of using the current data for calculation, more than 500 patients are needed to detect differences between groups; 2) there was female dominance, so the finding may not be applicable to both genders; and 3 ) the true control of intervention is difficult as a majority of patients are more likely to use their preferred rescue drugs and have a variety of physical activity.

\section{CONCLUSION}

The traditional Thai massage and joint mobilization used in this study were equally effective for short-term reduction of pain and disability in chronic nonspecific LBP. Both techniques were safe with short-term effect in a chosen group of patients. Patients should also be encouraged to take their own active roles in self-management as a part of their lives.

\section{CONFLICT OF INTEREST NOTIFICATION}

The authors declare there are no conflicts of interest.

\section{COPYRIGHT}

Published under the CreativeCommons AttributionNonCommercial-NoDerivs 3.0 License.

\section{REFERENCES}

1. Bakker EW, Verhagen AP, van Trijffel E, Lucas C, Koes BW. Spinal mechanical load as a risk factor for low back pain: a systematic review of prospective cohort studies. Spine. 2009;34(8):281-293.

2. Vandergrift JL, Gold JE, Hanlon A, Punnett L. Physical and psychosocial ergonomic risk factors for low back pain in automobile manufacturing workers. Occup Environ Med. 2012;69(1):29-34.

3. Clays E, De Bacquer D, Leynen F, Kornitzer M, Kittel F, De Backer G. The impact of psychosocial factors on low back pain: longitudinal results from the Belstress study. Spine. 2007;32(2):262-268.

4. Picavet HS, Schouten JS. Physical load in daily life and low back problems in the general population-The MORGEN study. Prev Med. 2000;31(5):506-512.

5. Deyo RA, Cherkin D, Conrad D, Volinn E. Cost, controversy, crisis: low back pain and the health of the public. Ann Rev Publ Health. 1991;12(1):141-156.

6. Frymoyer JW, Cats-Baril WL. An overview of the incidences and costs of low back pain. Orthop Clin N Am. 1991;22(2):263-271.

7. Furlan AD, Clarke J, Esmail R, Sinclair S, Irvin E, Bombardier C. A critical review of reviews on the treatment of chronic low back pain. Spine. 2001;26(7):E155-E162.

8. Lewis M, Johnson MI. The clinical effectiveness of therapeutic massage for musculoskeletal pain: a systematic review. Physiotherapy. 2006;92(3):146-158. 
9. Pengel HM, Maher CG, Refshauge KM. Systematic review of conservative interventions for subacute low back pain. Clin Rehabil. 2002;16(8):811-820.

10. Adams MH, White MS. The effects of massage therapy on pain management in the acute care setting. Int J Ther Massage Bodywork. 2010;3(1):4.

11. Kumar S, Beaton K, Hughes T. The effectiveness of massage therapy for the treatment of nonspecific low back pain: a systematic review of systematic reviews. Int J Gen Med. 2013;6:733-741.

12. Imamura $M$, Furlan $A D$, Dryden $T$, Irvin E. Evidence-informed management of chronic low back pain with massage. Spine $J$. 2008;8(1):121-133.

13. Bronfort G, Haas M, Evans RL, Bouter LM. Efficacy of spinal manipulation and mobilization for low back pain and neck pain: a systematic review and best evidence synthesis. Spine J. 2004;4(3):335-356.

14. Kirkaldy-Willis WH, Cassidy JD. Spinal manipulation in the treatment of low-back pain. Can Fam Physician. 1985;31:535.

15. Mackawan S, Eungpinichpong W, Pantumethakul R, Chatchawan U, Hunsawong T, Arayawichanon P. Effects of traditional Thai massage versus joint mobilization on substance $\mathrm{P}$ and pain perception in patients with non-specific low back pain. $J$ Bodyw Mov Ther. 2007;11(1):9-16.

16. Tapanya, S. Traditional Thai Massage. Bangkok: Duang Kamol; 1993.

17. Buttagat V, Eungpinichpong W, Chatchawan U, Kharmwan S. The immediate effects of traditional Thai massage on heart rate variability and stress-related parameters in patients with back pain associated with myofascial trigger points. J Bodyw Mov Ther. 2011;15(1):15-23.

18. Chatchawan U, Thinkhamrop B, Kharmwan S, Knowles J, Eungpinichpong W. Effectiveness of traditional Thai massage versus Swedish massage among patients with back pain associated with myofascial trigger points. J Bodyw Mov Ther. 2005;9(4):298-309.

19. Ryan C, Keiwkarnka B, Khan MI. Traditional Thai massage: unveiling the misconceptions and revealing the health benefits. J Public Health Development. 2003;1(2):69-75.

20. Cherkin DC, Sherman KJ, Kahn J, Wellman R, Cook AJ, Johnson E, et al. A comparison of the effects of 2 types of massage and usual care on chronic low back pain: a randomized, controlled trial. Ann Intern Med. 2011;155(1):1-9.

21. Preyde M. Effectiveness of massage therapy for subacute low-back pain: a randomized controlled trial. Can Med Assoc J. 2000;162(13):1815-1820.

22. Furlan AD, Imamura M, Dryden T, Irvin E. Massage for low back pain: an updated systematic review within the framework of the Cochrane Back Review Group. Spine. 2009;34(16):1669-1684.

23. Koes BW, van Tulder M, Lin CW, Macedo LG, McAuley J, Maher C. An updated overview of clinical guidelines for the management of non-specific low back pain in primary care. Eur Spine J. 2010;19(12):2075-2094.

24. Cedraschi C, Robert J, Goerg D, Perrin E, Fischer W, Vischer TL. Is chronic non-specific low back pain chronic? Definitions of a problem and problems of a definition. Br J Gen Pract. 1999;49(442):358-362.
25. Conway PH, Clancy C. Comparative-effectiveness researchimplications of the Federal Coordinating Council's report. $N$ Engl J Med. 2009;361(4):328-330.

26. Bartis K, Willan A. Intention-to-treat and per-protocol analysis. CMAJ. 2011;183(6): 696.

27. Maitland GD. Matland's vertebral manipulation, 7th ed. London, UK: Butterworth-Heinemann; 2005.

28. Beattie PF, Arnot CF, Donley JW, Noda H, Bailey L. The immediate reduction in low back pain intensity following lumbar joint mobilization and prone press-ups is associated with increased diffusion of water in the L5-S1 intervertebral disc. J Orthop Sports Phys Ther. 2010;40(5):256-264.

29. McKenzie RA. The lumbar spine: mechanical diagnosis and therapy. Christchurch, New Zealand: Spinal Publications; 1981.

30. Katz J, Rosenbloom BN. the golden anniversary of Melzack and Wall's gate control theory of pain: celebrating 50 years of pain research and management. Pain Res Manag. 2015;20(6): 285-286.

31. Van Tulder MW, Koes BW, Bouter LM. Conservative treatment of acute and chronic nonspecific low back pain: a systematic review of randomized controlled trials of the most common interventions. Spine. 1997;22(18):2128-2156.

32. Fritz JM, Hebert J, Koppenhaver S, Parent E. Beyond minimally important change: defining a successful outcome of physical therapy for patients with low back pain. Spine. 2009;34(25):2803-2809.

33. Triano JJ, McGregor M, Hondras MA, Brennan PC. Manipulative therapy versus education programs in chronic low back pain. Spine. 1995;20(8):948-955.

34. Fritz JM, Irrgang JJ. A comparison of a modified Oswestry Low Back Pain Disability Questionnaire and the Quebec Back Pain Disability Scale. Phys Ther. 2001;81(2):776-788.

35. Daltroy LH, Cats-Baril WL, Katz JN, Fossel AH, Liang MH. The North American Spine Society Lumbar Spine Outcome Assessment Instrument: reliability and validity tests. Spine. 1996;21(6):741-748.

36. George SZ, Robinson ME. Preference, expectation, and satisfaction in a clinical trial of behavioral interventions for acute and sub-acute low back pain. J Pain. 2010;11(11):1074-1082.

37. Helmhout PH, Staal JB, Heymans MW, Harts CC, Hendriks EJ, de Bie RA. Prognostic factors for perceived recovery or functional improvement in non-specific low back pain: secondary analyses of three randomized clinical trials. Eur Spine J. 2010;19(4):650-659.

38. Shiri R, Karppinen J, Leino-Arjas P, Solovieva S, ViikariJuntura E. The association between obesity and low back pain: a meta-analysis. Am J Epidemiol. 2009;171(2):135-154.

39. Chou R, Huffman LH. Nonpharmacologic therapies for acute and chronic low back pain: a review of the evidence for an American Pain Society/American College of Physicians clinical practice guideline. Ann Intern Med. 2007;147(7):492-504.

Corresponding author: Thavat Prasartritha, MD, Institute of Orthopaedics, Lerdsin Hospital, $190 \mathrm{Si}$ Lom, Silom, Bang Rak, Bankok 10500, Thailand E-mail: prasartritha1@hotmail.com 